\title{
Duration from seroconversion to eligibility for antiretroviral therapy and from ART eligibility to death in adult HIV-infected patients from low and middle-income countries: collaborative analysis of prospective studies
}

\author{
The eligibility for ART in lower income countries (eART-linc) collaboration
}

Correspondence to:

Dr Marcel Zwahlen, Institute of Social and Preventive Medicine (ISPM), University of Bern, Finkenhubelweg 11, CH-3012

Bern, Switzerland; eART-linc@ ispm.unibe.ch

See end of paper for members of writing group.

Accepted 13 May 2008

\section{UNLOCKI}

This paper is freely available online under the BMJ Journals unlocked scheme, see http:// sti.bmj.com/info/unlocked.dtl

\begin{abstract}
Background: Estimation of the number of people in need of antiretroviral therapy (ART) in resource-limited settings requires information on the time from seroconversion to ART eligibility and from ART eligibility to death.
\end{abstract}

Objectives: To estimate duration from seroconversion to different ART eligibility criteria and from ART eligibility to death in HIV-infected adults in low-income and middleincome countries.

Methods: Participants with documented seroconversion from five cohorts (two cohorts from Uganda, two from Thailand and one from Côte d'Ivoire) were analysed. We used Weibull survival models and Bayesian simulation methods to model true (unobserved) first time of treatment eligibility. We set a consistency constraint so that the mean duration from seroconversion to death was equal to the mean from seroconversion to ART eligibility plus the mean from eligibility to death.

Results: We analysed data from 2072 participants, 16157 person-years of follow-up and 794 deaths. For the criterion CD4 T-lymphocyte count $<200$ cells $\times 10^{6} /$, the median duration from seroconversion to ART eligibility was 6.1 years (95\% credibility interval 3.3-10.4) for all studies and 7.6 years (95\% credibility interval 3.4-15.2) for all but the Thai cohorts. Corresponding estimates for the time from CD4 T-lymphocyte count $<200$ cells $\times 10^{6} / 1$ to death were 2.1 years $(0.7-4.8)$ and 2.7 years $(0.8-$ 8.4). When including all cohorts, the mean time from serconversion to CD4 T-lymphocyte count $<200$ cells $\times 10^{6} / \mathrm{l}$ and from CD4 T-lymphocyte count $<200$ cells $\times 10^{6} / 1$ to death represented $66 \%(38-87 \%)$ and $34 \%$ (13-62\%), respectively of the total survival time.

Conclusions: The duration of different ART eligibility criteria to death was longer than the estimates used in previous calculations of the number of people needing ART. However, uncertainty in estimates was considerable and heterogeneity across cohorts important.

The scale-up of antiretroviral therapy (ART) represents an unprecedented effort to provide access to life-saving drugs to HIV-infected patients in resource-limited settings. The World Health Organization estimates that as a result two million people living with HIV/AIDS in low-income and middle-income countries were receiving treatment at the end of in $2006 .{ }^{1}$ An important question is the coverage of ART in these settings; in other words, what proportion of patients in need of ART is receiving it, and how far away from reaching the goal of universal access are we?

To estimate coverage of ART, information about the untreated natural history of HIV infection in low-income and middle-income countries is needed, especially on the duration from infection (seroconversion) to the point in time when an individual becomes eligible for ART, and from ART eligibility to death. ${ }^{23}$ Criteria for treatment eligibility differ between settings and guidelines. For example, the current International AIDS SocietyUSA guidelines for treatment of HIV infection in adults ${ }^{4}$ recommend that ART should be considered in asymptomatic patients after the CD4 count falls below 350 cells $\times 10^{6} / 1$ and before it declines to 200 cells $\times 10^{6} / 1$. In contrast, the $2002 \mathrm{WHO}$ guidelines, which are still used in some countries-for example, in South Africa, recommend ART only for patients with WHO stage 4 disease or a CD4 cell count of less than 200 cells $\times 10^{6} / 1$. $^{5}$ These recommendations were revised in 2003 and now state that, in addition, treatment should also be started in patients with WHO stage 3 and a CD4 count between 200 and 350 cells $\times 10^{6} / 1{ }^{6}$

Few seroconverter cohorts exist in low-income and middle-income countries, and the number of participants enrolled is relatively small. Collaborative analyses of individual participant data from several studies are useful in this situation. ${ }^{7}$ Recently, Todd and colleagues ${ }^{8}$ estimated time from seroconversion to death in a collaborative analysis of eight seroconverter cohorts from low-income and middle-income countries before ART became available; however, their study did not address time to ART eligibility. We established the eligibility for Antiretroviral Therapy in lower income countries (eART-linc) collaboration with the aim to estimate time from seroconversion to ART eligibility and from ART eligibility to death in HIV-infected adults from lower-income countries.

\section{METHODS}

\section{Cohort studies}

We contacted cohort studies from low-income and middle-income countries of HIV-infected adults with documented last negative and first positive HIV tests and information on the evolution of CD4 counts or clinical stage, and invited them to contribute data to the eART-linc collaboration. 
Five cohorts, which were established in the 1990s and described in detail elsewhere, ${ }^{9-13}$ participated in the current analyses (table 1): the Primo-CI ANRS 1220 cohort $^{9}$ in Abidjan, Côte d'Ivoire; the Rakai District cohort ${ }^{10}$ in rural Uganda; the Masaka District cohort, ${ }^{11}$ also in rural Uganda; the Royal Thai Army conscripts cohort ${ }^{12}$ in Thailand and the blood donors cohort ${ }^{13}$ in Chiang Mai, Thailand. Inspection of the data received showed that the information on CD4 count evolution was limited for the Thai cohorts, ${ }^{12}{ }^{13}$ and these therefore contributed to estimation of the time from seroconversion to death only. Also, information on clinical stage was available only for the Masaka cohort. ${ }^{11}$

\section{Observable and unobservable information}

Figure 1 shows the observable information and the time points and intervals we set out to estimate. The exact date of reaching treatment eligibility cannot be observed because the relevant criteria, including CD4 counts and clinical stage, are assessed at certain points in time only. Individuals for whom treatment eligibility was observed had two dates: the last date with available information before eligibility was observed and the first date at which available information indicated that ART eligibility had been reached. The true date of treatment eligibility will lie somewhere between these two dates.

\section{Statistical analyses}

We used Weibull survival models ${ }^{14}$ to estimate the duration from seroconversion to various eligibility criteria for ART, from ART eligibility to death or from serconversion to death. The date of seroconversion was calculated as the midpoint between the last documented negative HIV test and the first documented positive HIV test. Follow-up was censored on the date the patient was last known to be alive. In patients who initiated ART, follow-up was censored on the date of starting therapy. We did analyses for various CD4 thresholds $\left(<200\right.$ cells $\times 10^{6} / 1$, $<275$ cells $\times 10^{6} / 1,<350$ cells $\left.\times 10^{6} / 1\right)$ and for the combined criteria WHO clinical stage 3 and CD4 $<350$ cells $\times 10^{6} / 1$. In additional multivariable analyses we included gender and age. HIV subtype could not be included because subtype was highly correlated with the country in which the cohort was conducted. For descriptive purposes, we calculated and plotted KaplanMeier cumulative incidence estimates for some outcomes for each cohort.

We accounted for differences in survival distributions between cohorts using the hierarchical random effects model assuming proportional hazards. The random effects were assumed to be normally distributed around a common mean. We allowed for two different Weibull distributions, one each for the duration from seroconversion to treatment eligibility and from treatment eligibility to death. For the Thai cohorts, which did not contribute information on treatment eligibility, we modelled the duration from seroconversion to death using a third Weibull distribution. We set a consistency constraint on the mean survival times such that the mean duration from seroconversion to death was equal to the sum of the mean duration from seroconversion to the estimated time of ART eligibility and the mean duration from ART eligibility to death. In an additional analysis we excluded the Thai cohorts.

Estimation of time periods was based on a fully probabilistic approach using simulation methods. Vague prior distributions for parameters of interest were specified by using normal distributions with variances equal to $10^{3}$. Samples from the posterior distributions for quantities of interest were obtained by Markov Chain Monte Carlo simulations based on 100000 iterations from which the first 60000 iterations were discarded. The influence of age at seroconversion and sex on survival was explored in standard proportional hazard Weibull regression models. Age was entered as a continuous variable.

All analyses were performed using Stata (version 9.2, College Station, TX, USA) and WinBUGS ${ }^{15}$ (version 1.4.1, Cambridge, UK). Results are presented as means and medians with $95 \%$ credibility intervals (CrI) or as conventional 95\% confidence intervals (CI). In Bayesian statistics credibility intervals are commonly used to provide summary information about the probability distribution of a parameter of interest. Similar to 95\% confidence intervals, they cover a range in which the true value of the unknown quantity lies with $95 \%$ probability.

Table 1 Characteristics of cohorts and participants included in analyses

\begin{tabular}{|c|c|c|c|c|c|c|}
\hline & \multicolumn{5}{|l|}{ Cohort } & \multirow[b]{2}{*}{ Combined } \\
\hline & $\begin{array}{l}\text { Côte d'Ivoire } \\
\text { (Abidjan) }\end{array}$ & Uganda (Masaka) & Uganda (Rakai) & $\begin{array}{l}\text { Thailand (Royal } \\
\text { Thai Army) }\end{array}$ & Thailand (Chiang Mai) & \\
\hline Reference & Minga et $a l^{9}$ & Morgan et $a l^{32}$ & Wawer et al ${ }^{33}$ & Rangsin et $a l^{12}$ & Nagachinta et $a l^{13}$ & - \\
\hline Study population & Blood donors & $\begin{array}{l}\text { Participants in } \\
\text { community cohort }\end{array}$ & $\begin{array}{l}\text { Participants in } \\
\text { community cohort }\end{array}$ & $\begin{array}{l}\text { Military } \\
\text { conscripts }\end{array}$ & $\begin{array}{l}\text { Male blood donors and } \\
\text { their female partners }\end{array}$ & - \\
\hline Year of start of study & 1997 & 1990 & 1991 & 1998 & 1992 & \\
\hline $\begin{array}{l}\text { No of participants included in } \\
\text { analyses }(\%)\end{array}$ & $253(100)$ & $338(100)$ & $411(100)$ & $233(100)$ & $837(100)$ & $2072(100)$ \\
\hline $\begin{array}{l}\text { Median (IQR) interval between } \\
\text { last negative and first positive } \\
\text { HIV-1 test, months }\end{array}$ & $7.5(3.4-19.1)$ & $12.5(11.7-24.3)$ & $22.4(14.6-37.7)$ & $8.5(5.7-9.5)$ & $50.7(31.6-69.8)$ & $24.0(11.8-50.1)$ \\
\hline No of women $(\%)$ & $97(38.3)$ & $180(53.2)$ & $256(62.3)$ & $0(0)$ & $267(31.9)$ & $800(38.6)$ \\
\hline No of patients starting ART (\%) & $79(31.2)$ & 57 (16.9) & $151(36.7)$ & $0(0)$ & $0(0)$ & $287(13.8)$ \\
\hline $\begin{array}{l}\text { Mean (SD) age at baseline, } \\
\text { years }\end{array}$ & $29.1(7.1)$ & $32.0(13.6)$ & $35.8(2.5)$ & $22.1(0.9)$ & $28.7(6.4)$ & $29.9(8.3)$ \\
\hline $\begin{array}{l}\text { Median (IQR) baseline CD4 cell } \\
\text { count (IQR), cells } \times 10^{6} / /\end{array}$ & $472(331-648)$ & $553(425-743)$ & $409(235-584)$ & NA & NA & - \\
\hline $\begin{array}{l}\text { Median (IOR) number of CD4 } \\
\text { counts per person }\end{array}$ & $9(5-14)$ & $5(2-11)$ & $3(2-7)$ & NA & NA & - \\
\hline $\begin{array}{l}\text { Median (IOR) follow up from } \\
\text { seroconversion, years }\end{array}$ & $5.4(5.4-7.6)$ & $4.9(3.3-7.8)$ & $5.7(3.8-7.6)$ & $6.0(5.3-6.7)$ & $6.5(5.2-9.1)$ & $6.0(4.4-7.8)$ \\
\hline
\end{tabular}

NA, not systematically assessed and therefore not analysed in this study; SD, standard deviation; IQR, interquartile range.

The baseline CD4 count relates to the first measurement after seroconversion. 


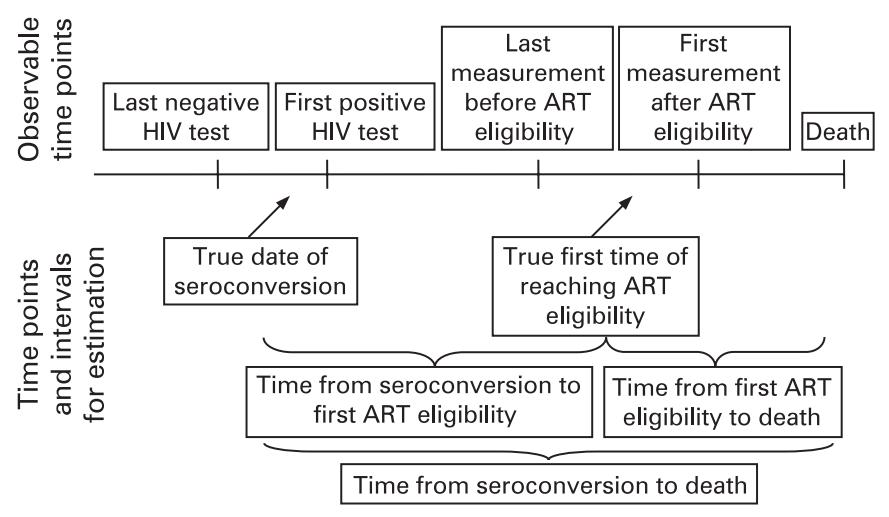

Figure 1 Schematic representation of observed and estimated time points and intervals in the natural history of HIV infection.

\section{RESULTS}

A total of 2072 adults with documented seroconversion were included in the analyses (table 1). In the African cohorts the follow-up of 294 patients (14.2\%) was censored because ART was started. In total, $794(38.3 \%)$ patients died, most of them (762, 96.0\%) while not on ART. Figure 2 shows the cumulative incidences from seroconversion to death or to first time ART eligibility was observed for different eligibility criteria. For time to ART eligibility the figure also includes the fitted curves based on the median of the posterior distribution of the common mean of the Weibull parameters. Owing to the difference between the true (but unobservable) time of reaching ART eligibility for the first time, and the first time eligibility was observed, the fitted curves are generally above the observed curves.

Table 2 shows the estimated mean and median years from seroconversion to different ART eligibility criteria and from ART eligibility to death. Means were 1-2 years higher than medians and ranged from 7.6 years from seroconversion to a CD4 count $<200$ cells $\times 10^{6} / 1$ to 3.9 years for the period from reaching a CD4 count $<200$ cells $\times 10^{6} / 1$ to death. Corresponding medians were 6.1 years and 2.1 years. For most estimates $95 \%$ credibility intervals were fairly wide. The additional analysis excluding the Thai cohorts resulted in intervals that were 0.5 to 1.6 years longer for medians and 0.8 to 2.1 years longer for means, with substantially wider confidence intervals. For the CD4 criteria, the sum of the mean duration from seroconversion to eligibility and the mean duration from eligibility to death gave mean durations from seroconversion to death of 11.3-11.7 years, depending on the eligibility criterion (table 2). Excluding the Thai cohorts resulted in longer periods, with a sum of the means of the two periods ranging from 13.7 years to 14.6 years (table 2). The sum of the means for the combined CD4 and clinical stage criterion, which was estimated using the Masaka cohort data only, was 10.9 years. Note that the medians shown in table 2 cannot simply be added to obtain the median duration from seroconversion to death, only the means add up in this way. To increase applicability to different settings, table 2 also gives time intervals expressed as the percentage of the total time from seroconversion to death.

Heterogeneity of model parameters across cohorts was important. The mean of the random effects standard deviation ranged from 0.8 to 0.92 with lower credibility bounds above 0.4 . When including age and sex in the models, estimates of survival were reduced for older people, with hazard ratios ranging from 1.02 to 1.03 per year increase in age with lower bounds of the $95 \%$ credibility intervals 1.01 and upper bounds not exceeding 1.05. Analyses comparing women with men gave hazard ratios ranging from 0.91 to 1.09 with wide $95 \%$ credibility intervals that always included 1: lower $95 \%$ credibility bounds ranged from 0.62 to 0.71 , higher bounds from 1.4 to 1.7
Figure 2 Kaplan-Meier plots of the cumulative probability of death and of reaching different eligibility criteria for antiretroviral therapy.

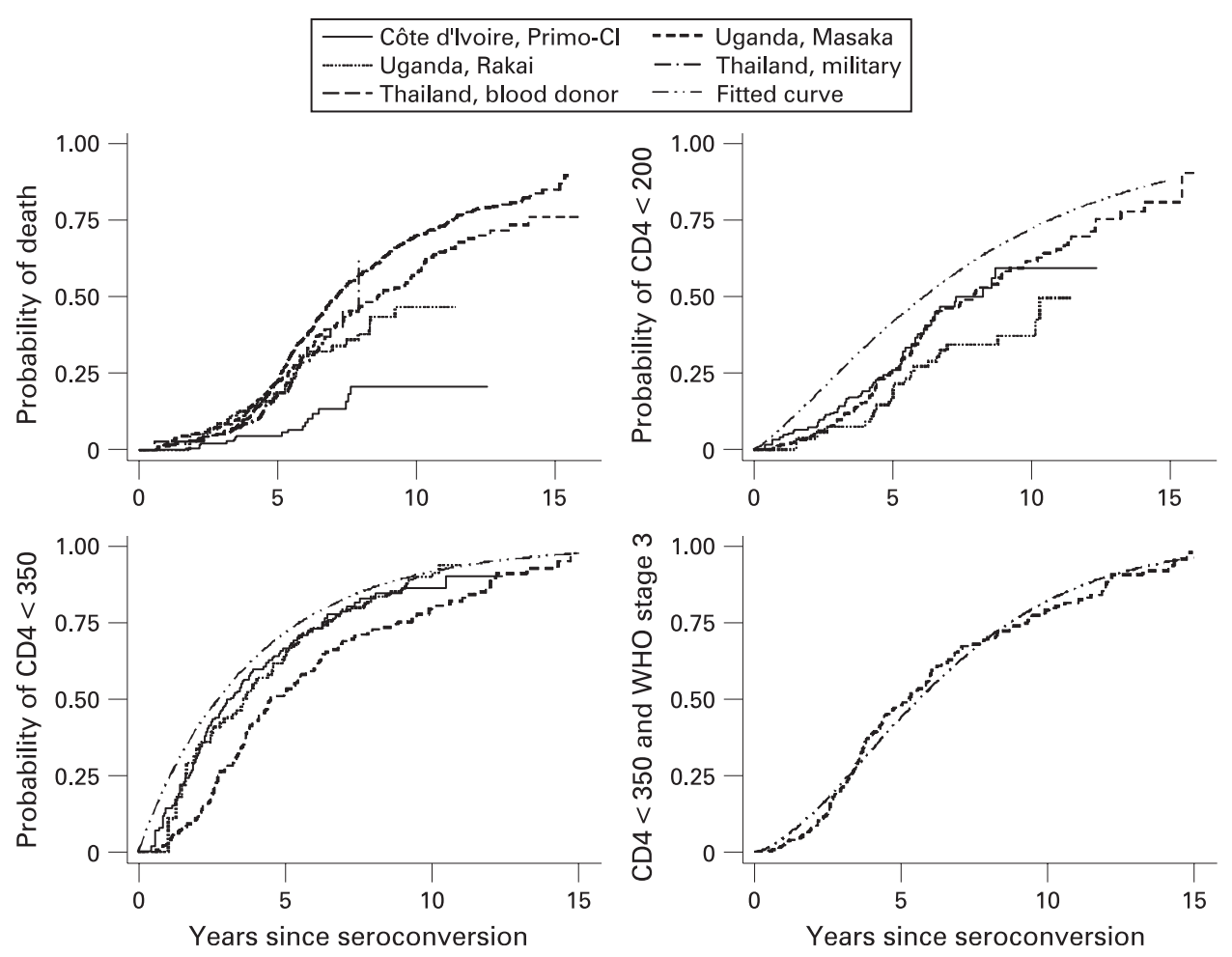


Table 2 Estimated time from seroconversion to different ART eligibility criteria and from ART eligibility to death

\begin{tabular}{|c|c|c|c|c|}
\hline \multirow[b]{2}{*}{ Time interval } & \multicolumn{4}{|l|}{ ART eligibility criteria } \\
\hline & $<200$ CD4 cells $\times 10^{6} / I$ & $<275$ CD4 cells $\times 10^{6} / I$ & $<350$ CD4 cells $\times 10^{6} / 1$ & $\begin{array}{l}<350 \text { CD } 4 \text { cells } \times 10^{6} / I \text { and } \\
\text { WHO stage } 3\end{array}$ \\
\hline \multicolumn{5}{|l|}{ All cohorts } \\
\hline Median (95\% Crl) & $6.1(3.3-10.4)$ & $4.2(1.9-7.9)$ & $2.7(0.9-6.3)$ & $5.6(5.0-6.2)$ \\
\hline Mean (95\% Crl) & $7.6(4.1-12.9)$ & $5.4(2.5-10.3)$ & $4.0(1.4-9.2)$ & $6.4(5.8-7.1)$ \\
\hline Percentage ${ }^{*}$ & $66(38-87)$ & $48(23-75)$ & $34(12-64)$ & $58(50-65)$ \\
\hline Median (95\% Crl) & $2.1(0.7-4.8)$ & $4.0(1.7-8.0)$ & $6.0(2.9-11.7)$ & $3.0(2.4-3.8)$ \\
\hline Mean (95\% Crl) & $3.9(1.4-9.1)$ & $5.9(2.5-11.7)$ & $7.7(3.8-15.0)$ & $4.5(3.5-6.4)$ \\
\hline Percentage ${ }^{*}$ & $34(13-62)$ & $52(25-78)$ & $66(36-88)$ & $42(35-50)$ \\
\hline \multicolumn{5}{|c|}{ Excluding the two Thai cohorts } \\
\hline \multicolumn{5}{|c|}{ Seroconversion to eligibility (years) } \\
\hline Median (95\% Crl) & $7.6(3.4-15.2)$ & $4.8(1.9-11.6)$ & $3.2(1.0-9.7)$ & $5.6(5.1-6.2)$ \\
\hline
\end{tabular}

*Percentage of duration from seroconversion to death.

$\mathrm{Crl}$, credibility interval.

\section{DISCUSSION}

The eART-linc collaboration, which currently includes over 2000 untreated adults from lower-income settings with documented seroconversion, made analyses of individual participant data to estimate the periods from seroconversion to different ART eligibility criteria, and from ART eligibility to death possible.

The collaborative analysis of individual participant data is an important strength of this study: meta-analyses that use individual participant data are considered the gold standard and yardstick for meta-analyses and systematic reviews. ${ }^{16}$ Indeed, in previous reviews of published studies ${ }^{17}{ }^{18}$ we found that the type of data presented in articles was very heterogeneous, confirming earlier observations on common difficulties with systematic reviews of prognostic ${ }^{19}$ and other observational studies. This approach is also used by the Alpha Network (Analysing Longitudinal Population-based HIV/Aids data on Africa) to analyse patterns of mortality in HIV-infected adults, based on pooled data from several community-based cohort studies. ${ }^{8} 2021$

The exact date of reaching treatment eligibility can usually not be observed because the relevant criteria, including CD4 counts and clinical stage, are not assessed continuously. One approach to this problem is to take the first date eligibility criteria are met, which may provide a good approximation if the relevant information is collected regularly and frequently. However, this is problematic in the more common situation when intervals between measurements are relatively wide, leading to underestimation of the duration from true ART eligibility to death. In clinical decision-making the date the criteria for ART are documented for the first time is the important date and should lead to the initiation of ART. Of note, many patients in lower-income countries start ART late or very late, with CD4 cell counts well below 200 cells $\times 10^{6} / 1 .^{22-24}$ However, when estimating the need at the population level, it is the actual date of having reached ART eligibility for the first time that is of interest. In the present analysis we chose an analytical strategy that took into account that only interval information on ART eligibility was available from cohorts. Unsurprisingly, our estimates are higher than published estimates, which took the date when eligibility was documented for the first time as the point of departure. For example, we estimated the median duration from a CD4 count $<200$ cells $\times 10^{6} / 1$ to death as 2.1 years $(2.7$ years when excluding the two cohorts from Thailand) and the mean as 3.9 years (5.2 years when excluding the two cohorts from Thailand). Previously published estimates in untreated patients range from 7 months to 22 months (table 3 ). The distribution of survival times is skewed, which explains that estimates for the mean were higher than those for medians.

Mortality patterns after seroconversion have been previously described for several of the seroconverter cohorts included in this analysis. ${ }^{20}{ }^{25-29}$ Interestingly, mortality was similar across cohorts, particularly in the first years after seroconversion,

Table 3 Published estimates of survival from CD lymphocyte counts $<200$ CD4 cells $\times 10^{6} /$ I

\begin{tabular}{|c|c|c|c|c|}
\hline Study, year of publication & No & Country & Years & $\begin{array}{l}\text { Median months of survival } \\
(95 \% \mathrm{CI})\end{array}$ \\
\hline Lawn, $2005^{34}$ & 134 & South Africa & $2002-5$ & 20 (14 to 28 ) \\
\hline Costello, $2005^{35}$ & 167 & Thailand & $1993-9$ & $21.6(19 \text { to } 24)^{*}$ \\
\hline Badri, $2004^{36}$ & 447 & South Africa & 1992-2001 & 23.6 (21 to 27$)$ \\
\hline Schim van der Loeff, $2002^{37}$ & 378 & The Gambia & $1986-97$ & 7 (5 to 9$)$ \\
\hline Kilmarx, $2000^{38}$ & 15 & Thailand & $1991-8$ & 11 (7 to 15$)$ \\
\hline French, $1999^{39}$ & 78 & Uganda & before 1998 & $9(7$ to 15$)$ \\
\hline Van der Paal, $2007^{28}$ & 147 & Uganda & 1990-2003 & 25.7 (21 to 31$)$ \\
\hline
\end{tabular}

*Men only.

$\mathrm{Cl}$, confidence interval. 
despite differences in HIV subtypes. One exception was the cohort from Côte d'Ivoire, which showed lower cumulative mortality than the other four cohorts, despite similar probabilities of observing CD4 criteria for ART eligibility. The lower mortality and morbidity might be explained by under-ascertainment of deaths in this clinic-based cohort or by the early introduction of cotrimoxazole prophylaxis, in 1994, by the Centre National de Transfusion Sanguine in Abidjan. ${ }^{9}$ Indeed, two clinical trials from Côte d'Ivoire and one from Senegal showed a substantial reduction of mortality: the combined relative risk was 0.69 (95\% confidence interval 0.55 to 0.87$).{ }^{30}$

About five years after seroconversion mortality was higher in the Thai studies and, particularly, in the Chiang Mai blood donor cohort. ${ }^{13}$ The inclusion of paid blood donors of lower socioeconomic strata might explain the higher mortality in this cohort. There is debate on whether HIV subtype (predominantly subtype $\mathrm{E}$ in Thailand) influences survival. ${ }^{8}$ The additional analysis excluding the Thai studies therefore resulted in longer duration estimates. In addition to reporting time intervals in years, we therefore also expressed intervals as the percentage of the total time from seroconversion to death. This should facilitate the application of results to populations with different background and HIV-related mortality. We found that depending on the ART eligibility criteria considered, 34\% to $66 \%$ of the mean duration from seroconversion to death occurs after having become eligible for ART.

Our analysis has several limitations. The number of included cohorts and the number of seroconverters was small and information on treatment eligibility was available for only three of the five cohorts. We used a simple midpoint approximation to estimate the date of seroconversion, despite the fact that in some cohorts intervals between tests were long. Estimation of model parameters became difficult when allowing uncertainty about the date of seroconversion as well as the time of reaching ART eligibility. Also, midpoint approximation is common practice in analyses of survival time from seroconversion to death. We analysed all-cause mortality and could not distinguish between HIV-associated mortality and other causes of death. We used parametric survival models and thus made assumptions about the shape of the survival distributions that may not always be appropriate.

In conclusion, estimation of the number of people in need of ART in resource-limited settings should be based on figures for survival from ART eligibility to death that are somewhat higher than those used previously, ${ }^{2}$ and support the recent updates to the Joint United Nations Programme on HIV/AIDS (UNAIDS) Spectrum projection software. ${ }^{31}$ Finally, we would welcome additional studies in the eART-linc collaboration in order to obtain more precise and more widely applicable estimates in future analyses. Interested investigators are invited to contact us at eART-linc@ispm.unibe.ch.

Acknowledgements: We are grateful to Peter Ghys without whom the eART-linc collaboration would not exist. The analyses presented in this paper were supported by UNAIDS.

\section{Competing interests: None.}

The eART-linc collaboration writing group: Simon Wandel, ${ }^{1}$ Matthias Egger, ${ }^{1,2}$ Ram Rangsin, ${ }^{3}$ Kenrad E Nelson, ${ }^{4}$ Caroline Costello, ${ }^{5}$ Charlotte Lewden, ${ }^{6,7}$ Tom Lutalo, ${ }^{8}$ Anthony Ndyanabo, Jim Todd, ${ }^{9}$ Lieve Van der Paal, ${ }^{9}$ Albert Minga, ${ }^{10}$ Marcel Zwahlen ${ }^{1}$

${ }^{1}$ Institute of Social and Preventive Medicine (ISPM), University of Bern, Switzerland; ${ }^{2}$ Department of Social Medicine, University of Bristol, UK; ${ }^{3}$ Department of Military and Community Medicine, Phramongkutklao College of Medicine, Thailand; ${ }^{4}$ Department of Epidemiology, Johns Hopkins University, Baltimore, MD, USA; ${ }^{5}$ Department of Epidemiology, University of California, Los Angeles, CA, USA; ${ }^{6}$ INSERM, U593, Bordeaux, France; 'Institut de Santé Publique, d?Epidémiologie et de Developpement
(ISPED), Université Victor Segalen, Bordeaux, France; ${ }^{8}$ Rakai Health Sciences Program, Uganda Virus Research Institute, Entebbe, Uganda; ${ }^{9} \mathrm{MRC} /$ UVRI Uganda Research Unit on AIDS, Entebbe, Uganda; ${ }^{10}$ Programme PAC-Cl, CNTS, Abidjan, Côte d'Ivoire.

\section{REFERENCES}

1. World Health Organization. Antiretroviral therapy for HIV Infection in adults and adolescents in resource-limited settings: towards universal access. Geneva: WHO, 2006.

2. Boerma JT, Stanecki KA, Newell ML, et al. Monitoring the scale-up of antiretroviral therapy programmes: methods to estimate coverage. Bull World Health Organ 2006;84:145-50.

3. Stover J, Bertozzi S, Gutierrez JP, et al. The global impact of scaling up HIV/AIDS prevention programs in low- and middle-income countries. Science 2006;311:14746.

4. Hammer SM, Saag MS, Schechter M, et al. Treatment for adult HIV infection: 2006 recommendations of the International AIDS Society_-USA panel. Top HIV Med 2006;14:827-43.

5. World Health Organization. Scaling up antiretroviral therapy in ressource-limited settings: guidelines for a public health approach. Geneva: WHO, 2002.

6. World Health Organization. Scaling up antiretroviral therapy in ressource-limited settings: treatment guidelines for a public health approach. 2003 revision. Geneva: WHO, 2004.

7. Stewart LA, Tierney JF. To IPD or not to IPD? Advantages and disadvantages of systematic reviews using individual patient data. Eval Health Prof 2002;25:76-97.

8. Todd J, Glynn JR, Marston M, et al. Time from HIV seroconversion to death: a collaborative analysis of eight studies in six low and middle-income countries before highly active antiretroviral therapy. AIDS 2007;21(Suppl 6):S55-63.

9. Minga A, Danel C, Abo Y, et al. Progression to WHO criteria for antiretroviral therapy in a 7-year cohort of adult HIV-1 seroconverters in Abidjan, Cote d'Ivoire. Bull World Health Organ 2007;85:116-23.

10. Wawer MJ, Serwadda D, Gray RH, et al. Trends in HIV-1 prevalence may not reflect trends in incidence in mature epidemics: data from the Rakai population-based cohort, Uganda. AIDS 1997;11:1023-30.

11. Morgan D, Malamba SS, Maude GH, et al. An HIV-1 natural history cohort and survival times in rural Uganda. AIDS 1997;11:633-40.

12. Rangsin R, Chiu J, Khamboonruang $\mathrm{C}$, et al. The natural history of HIV-1 infection in young Thai men after seroconversion. J Acquir Immune Defic Syndr 2004;36:622-9.

13. Nagachinta T, Duerr A, Suriyanon V, et al. Risk factors for HIV-1 transmission from HIV-seropositive male blood donors to their regular female partners in northern Thailand. AIDS 1997;11:1765-72.

14. Lee ET. Statistical methods for survival data analysis. New York: John Wiley, 1992

15. Lunn DJ, Thomas A, Best N, et al. WinBUGS - a Bayesian modelling framework: concepts, structure, and extensibility. Statistics and Computing 2000;10:325-37.

16. Stewart LA, Parmar MK. Meta-analysis of the literature or of individual patient data: is there a difference? Lancet 1993;341:418-22.

17. Schneider M, Zwahlen M, Egger M. Natural history and mortality in HIV-positive individuals living in resource-poor settings: a literature review. UNAIDS Obligation HO/ 03/463871. Geneva: Joint United Nations Programme on HIV/AIDS (UNAIDS), 2005.

18. Zwahlen M, Egger M. Progression and mortality of untreated HIV-positive individuals living in resource-limited settings: update of literature review and evidence synthesis Geneva: UNAIDS, 2006.

19. Altman DG. Systematic reviews of evaluations of prognostic variables. BMJ 2001;323:224-8.

20. Zaba B, Marston M, Crampin AC, et al. Age-specific mortality patterns in HIVinfected individuals: a comparative analysis of African community study data. AIDS 2007;21(Suppl 6):S87-96.

21. Ghys PD, Zaba B, Prins M. Survival and mortality of people infected with HIV in low and middle income countries: results from the extended ALPHA network. AIDS 2007;21(Suppl 6):S1-4.

22. Dabis F, Balestre E, Braitstein P, et al. Cohort Profile: Antiretroviral Therapy in Lower Income Countries (ART-LINC): international collaboration of treatment cohorts. Int J Epidemiol 2005;34:979-86.

23. Braitstein P, Brinkhof MW, Dabis F, et al. Mortality of HIV-1-infected patients in the first year of antiretroviral therapy: comparison between low-income and high-income countries. Lancet 2006;367:817-24.

24. The ART-LINC Collaboration of the International Databases to Evaluate AIDS (IeDEA). Antiretroviral therapy in resource-limited settings 1996 to 2006: patient characteristics, treatment regimens and monitoring in sub-Saharan Africa, Asia and Latin America. Trop Med Int Health 2008;Mar 25 [Epub ahead of print].

25. Marston M, Todd J, Glynn JR, et al. Estimating 'net' HIV-related mortality and the importance of background mortality rates. AIDS 2007;21(Suppl 6):S65-71.

26. Nelson KE, Costello C, Suriyanon V, et al. Survival of blood donors and their spouses with HIV-1 subtype E (CRF01 A_E) infection in northern Thailand, 1992-2007. AIDS 2007;21(Suppl 6):S47-54.

27. Rangsin R, Piyaraj P, Sirisanthana $T$, et al. The natural history of HIV-1 subtype E infection in young men in Thailand with up to 14 years of follow-up. AIDS 2007;21(Suppl 6):S39-46.

28. Van der Paal L, Shafer LA, Todd J, et al. HIV-1 disease progression and mortality before the introduction of highly active antiretroviral therapy in rural Uganda. AIDS 2007;21(Suppl 6):S21-9. 
29. Lutalo T, Gray RH, Wawer M, et al. Survival of HIV-infected treatment-naive individuals with documented dates of seroconversion in Rakai, Uganda. AIDS 2007;21(Suppl 6):S15-9.

30. Grimwade $\mathbf{K}$, Swingler G. Cotrimoxazole prophylaxis for opportunistic infections in adults with HIV. Cochrane Database Syst Rev 2003;CD003108.

31. Stover J, Johnson P, Zaba B, et al. The Spectrum projection package: improvements in estimating mortality, ART needs, PMTCT impact and uncertainty bounds. Sex Transm Infect 2008;84(Suppl 1):i24-i30.

32. Morgan D, Maude GH, Malamba SS, et al. HIV-1 disease progression and AIDSdefining disorders in rural Uganda. Lancet 1997;350:245-50.

33. Sewankambo NK, Gray RH, Ahmad S, et al. Mortality associated with HIV infection in rural Rakai District, Uganda. AIDS 2000;14:2391-400.

34. Lawn SD, Myer L, Orrell C, et al. Early mortality among adults accessing a community-based antiretroviral service in South Africa: implications for programme design. AIDS 2005;19:2141-8.
35. Costello C, Nelson KE, Suriyanon V, et al. HIV-1 subtype E progression among northern Thai couples: traditional and non-traditional predictors of survival. Int J Epidemiol 2005;34:577-84.

36. Badri M, Bekker LG, Orrell C, et al. Initiating highly active antiretroviral therapy in sub-Saharan Africa: an assessment of the revised World Health Organization scalingup guidelines. AIDS 2004;18:1159-68.

37. Schim van der Loeff MF, Jaffar S, Aveika AA, et al. Mortality of HIV-1, HIV-2 and HIV-1/HIV-2 dually infected patients in a clinic-based cohort in the Gambia. AIDS 2002;16:1775-83.

38. Kilmarx PH, Limpakarnjanarat K, Kaewkungwal J, et al. Disease progression and survival with human immunodeficiency virus type 1 subtype $E$ infection among female sex workers in Thailand. J Infect Dis 2000;181:1598-606.

39. French N, Mujugira A, Nakiyingi J, et al. Immunologic and clinical stages in HIV-1infected Ugandan adults are comparable and provide no evidence of rapid progression but poor survival with advanced disease. J Acquir Immune Defic Syndr 1999;22:509-16. 